Copyright by the IOP PUBLISHING LTD. J. A. Calanog et al. 2013. "herMES: the far-infrared emission from dust-obscured galaxies," ApJ 77561 doi:10.1088/0004-637X/775/1/61

The Astrophysical Journal, 775:61 (10pp), 2013 September 20

doi:10.1088/0004-637X/775/1/61

(c) 2013. The American Astronomical Society. All rights reserved. Printed in the U.S.A.

\title{
HerMES: THE FAR-INFRARED EMISSION FROM DUST-OBSCURED GALAXIES
}

\author{
J. A. Calanog ${ }^{1}$, J. Wardlow ${ }^{1}$, Hai Fu ${ }^{1,2}$, A. Cooray ${ }^{1,3}$, R. J. Assef ${ }^{4}$, J. Bock ${ }^{3,4}$, C. M. Casey ${ }^{5}$, A. Conley ${ }^{6}$, D. Farrah ${ }^{7,8}$, \\ E. Ibar $^{9}$, J. Kartaltepe ${ }^{10}$, G. Magdis ${ }^{11}$, L. Marchetti ${ }^{12,13}$, S. J. Oliver ${ }^{7}$, I. PéreZ-Fournon ${ }^{14,15}$, D. Riechers ${ }^{3}$, \\ D. Rigopoulou ${ }^{11,16}$, I. G. Roseboom ${ }^{7,17}$, B. Schulz ${ }^{3,18}$, Douglas ScotT $^{19}$, M. SYmeonidis ${ }^{20}$, M. Vaccari ${ }^{13,21}$, \\ M. VIERO ${ }^{3}$, AND M. ZEMCOV ${ }^{3,4}$ \\ ${ }^{1}$ Department of Physics and Astronomy, University of California, Irvine, CA 92697, USA \\ ${ }^{2}$ Department of Physics and Astronomy, University of Iowa, Van Allen Hall, Iowa City, IA 52242, USA \\ ${ }^{3}$ California Institute of Technology, 1200 E. California Boulevard, Pasadena, CA 91125 , USA \\ ${ }^{4}$ Jet Propulsion Laboratory, 4800 Oak Grove Drive, Pasadena, CA 91109, USA \\ ${ }^{5}$ Institute for Astronomy, University of Hawaii, 2680 Woodlawn Drive, Honolulu, HI 96822, USA \\ ${ }^{6}$ Center for Astrophysics and Space Astronomy 389-UCB, University of Colorado, Boulder, CO 80309, USA \\ ${ }^{7}$ Astronomy Centre, Department of Physics and Astronomy, University of Sussex, Brighton BN1 9QH, UK \\ ${ }^{8}$ Department of Physics, Virginia Tech, Blacksburg, VA 24061, USA \\ ${ }^{9}$ Instituto de Astrofísica, Facultad de Física, Pontificia Universidad Católica de Chile, Casilla 306, Santiago 22, Chile \\ ${ }^{10}$ National Optical Astronomy Observatory, 950 North Cherry Avenue, Tucson, AZ 85719, USA \\ ${ }^{11}$ Department of Astrophysics, Denys Wilkinson Building, University of Oxford, Keble Road, Oxford OX1 3RH, UK \\ ${ }^{12}$ Department of Physical Sciences, The Open University, Milton Keynes MK7 6AA, UK \\ ${ }^{13}$ Dipartimento di Fisica e Astronomia, Università di Padova, vicolo Osservatorio, 3, I-35122 Padova, Italy \\ ${ }^{14}$ Instituto de Astrofísica de Canarias (IAC), E-38200 La Laguna, Tenerife, Spain \\ ${ }^{15}$ Departamento de Astrofísica, Universidad de La Laguna (ULL), E-38205 La Laguna, Tenerife, Spain \\ ${ }^{16}$ RAL Space, Rutherford Appleton Laboratory, Chilton, Didcot, Oxfordshire OX11 0QX, UK \\ ${ }^{17}$ Institute for Astronomy, University of Edinburgh, Royal Observatory, Blackford Hill, Edinburgh EH9 3HJ, UK \\ ${ }^{18}$ Infrared Processing and Analysis Center, MS 100-22, California Institute of Technology, JPL, Pasadena, CA 91125, USA \\ ${ }^{19}$ Department of Physics and Astronomy, University of British Columbia, 6224 Agricultural Road, Vancouver, BC V6T 1Z1, Canada \\ ${ }^{20}$ Mullard Space Science Laboratory, University College London, Holmbury St. Mary, Dorking, Surrey RH5 6NT, UK \\ ${ }^{21}$ Astrophysics Group, Physics Department, University of the Western Cape, Private Bag X17, 7535 Bellville, Cape Town, South Africa \\ Received 2013 April 16; accepted 2013 July 22; published 2013 September 5
}

\begin{abstract}
Dust-obscured galaxies (DOGs) are an ultraviolet-faint, infrared-bright galaxy population that reside at $z \sim 2$ and are believed to be in a phase of dusty star-forming and active galactic nucleus (AGN) activity. We present far-infrared (far-IR) observations of a complete sample of DOGs in the $2 \mathrm{deg}^{2}$ of the Cosmic Evolution Survey. The 3077 DOGs have $\langle z\rangle=1.9 \pm 0.3$ and are selected from $24 \mu \mathrm{m}$ and $r^{+}$observations using a color cut of $r^{+}-[24] \geqslant 7.5$ $\left(\mathrm{AB} \mathrm{mag}\right.$ ) and $S_{24} \geqslant 100 \mu \mathrm{Jy}$. Based on the near-IR spectral energy distributions, $47 \%$ are bump DOGs (star formation dominated) and $10 \%$ are power-law DOGs (AGN-dominated). We use SPIRE far-IR photometry from the Herschel Multi-tiered Extragalactic Survey to calculate the IR luminosity and characteristic dust temperature for the $1572(51 \%)$ DOGs that are detected at $250 \mu \mathrm{m}(\geqslant 3 \sigma)$. For the remaining $1505(49 \%)$ that are undetected, we perform a median stacking analysis to probe fainter luminosities. Herschel-detected and undetected DOGs have average luminosities of $(2.8 \pm 0.4) \times 10^{12} L_{\odot}$ and $(0.77 \pm 0.08) \times 10^{12} L_{\odot}$, and dust temperatures of $(33 \pm 7) \mathrm{K}$ and $(37 \pm 5) \mathrm{K}$, respectively. The IR luminosity function for DOGs with $S_{24} \geqslant 100 \mu \mathrm{Jy}$ is calculated, using far-IR observations and stacking. DOGs contribute $10 \%-30 \%$ to the total star formation rate (SFR) density of the universe at $z=1.5-2.5$, dominated by $250 \mu \mathrm{m}$ detected and bump DOGs. For comparison, DOGs contribute $30 \%$ to the SFR density for all $z=1.5-2.5$ galaxies with $S_{24} \geqslant 100 \mu \mathrm{Jy}$. DOGs have a large scatter about the star formation main sequence and their specific SFRs show that the observed phase of star formation could be responsible for their total observed stellar mass at $z \sim 2$.
\end{abstract}

Key words: galaxies: luminosity function, mass function - galaxies: star formation - infrared: galaxies

Online-only material: color figures

\section{INTRODUCTION}

The far-infrared (far-IR) luminosities of luminous infrared galaxies (LIRGs; $L_{\mathrm{IR}} \geqslant 10^{11} L_{\odot}$ ) and ultra-LIRGs (ULIRGs; $L_{\mathrm{IR}} \geqslant 10^{12} L_{\odot}$ ) are dominated by reprocessed thermal dust emission, due to a combination of star formation and active galactic nucleus (AGN) activity, with star formation typically being the more dominant component (e.g., Watabe et al. 2009; Elbaz et al. 2010). Locally, these sources are rare, although out to $z \sim 1$ they become more numerous and increasingly dominate the IR luminosity function of galaxies with increasing redshift (e.g., Le Floc'h et al. 2005; Pérez-González et al. 2005; Caputi et al. 2007; Magnelli et al. 2009; Rodighiero et al. 2010; Eales et al. 2010). (U)LIRGs are thought to trace a phase of intense star formation activity, which is likely followed by, or partially concurrent with, an episode of vigorous black hole accretion. It is postulated that upon the cessation of these phases, each produces an early-type galaxy (Genzel et al. 2001; Farrah et al. 2003; Lonsdale et al. 2006; Veilleux et al. 2009).

Studies using the Multiband Imaging Photometer for Spitzer (MIPS; Rieke et al. 2004) instrument on board the Spitzer Space Telescope (Werner et al. 2004) have identified high-redshift ULIRGs from their $24 \mu \mathrm{m}$ emission (e.g., Yan et al. 2004; Houck et al. 2005; Weedman et al. 2006; Fiore et al. 2008; Dey et al. 2008; Farrah et al. 2008; Fiore et al. 2009). Dey et al. (2008) exploited this technique in the Boötes field of the NOAO 
Deep Wide Field Survey and presented a sample of ULIRGs selected by the color cut $R-[24] \geqslant 14$ (Vega magnitudes; $\left.S_{24} / S_{R} \geqslant 1000\right)$. Applying this selection scheme effectively identifies high-redshift IR luminous galaxies containing large amounts of dust obscuration, which would be absent from ultraviolet (UV) selected samples. This color selection preferentially identifies the rest-frame $7.7 \mu \mathrm{m}$ polycyclic aromatic hydrocarbon feature found in star-forming galaxies and causes the redshift distribution to have a biased average at $z \sim 2$. Also, at $z \sim 2$, the dust-obscured galaxy (DOG) selection falls within range of the power-law component of AGN emission in the midIR, which also identifies a population of AGNs. It is proposed that these DOGs are the latter stage of the submillimeter galaxy (SMGs; Hughes et al. 1998; Smail et al. 1997; Barger et al. 1998; Blain et al. 1999, among others) phase where an AGN is triggered while star formation is still occurring, causing some dust to be heated to higher temperatures (Dey et al. 2008) than in classic $850 \mu \mathrm{m}$ selected SMGs. Pope et al. (2008) found that $30 \%$ of the SMGs are also DOGs, and of those SMG-DOGs, $30 \%$ are $\mathrm{AGN}$-dominated $(\geqslant 50 \%$ AGN contribution in mid-IR), consistent with this scenario. Using high-resolution optical and near-IR imaging from the Hubble Space Telescope to investigate DOG morphology, the studies of Bussmann et al. (2009, 2011) found that the morphologies of bump (star-forming) DOGs, power-law (AGN-dominated) DOGs, SMGs, and high-redshift quiescent distant red galaxies are consistent with the picture that major merger-driven systems eventually all evolve into compact relaxed passive galaxies (Springel et al. 2005 and references therein). Furthermore, Narayanan et al. (2010) used $N$-body and hydrodynamic simulations to model the temporal evolution of high-redshift galaxies and found that at the peak of the merger-driven galaxies' star formation rate (SFR), a galaxy can be identified as both an SMG and a DOG. The same study also found that during the stages after final coalescence, mergerdriven DOGs transition from being star formation dominated to being AGN dominated.

The launch of the Herschel Space Observatory ${ }^{22}$ (Pilbratt et al. 2010) enables the direct observation of DOGs in the far-IR regime, instead of extrapolating from spectral energy distribution (SED) templates or stacking (e.g., Dey et al. 2008; Pope et al. 2008). Melbourne et al. (2012) studied Herscheldetected DOGs with spectroscopic redshifts and showed that DOGs classified by their near-IR SEDs as either bump (starforming) or power-law (AGN-dominated) have $250 \mu \mathrm{m} / 24 \mu \mathrm{m}$ flux density ratios that are consistent with local ULIRGs of the respective classes. Penner et al. (2012) used Herschel data to show that DOGs' high rest-frame mid-infrared/UV flux density ratios are due to varying amounts of UV dust obscuration, and speculated that it is caused by differing degrees of alignment between dust and stars, or simply by the differences in total dust content.

The focus of this paper is to extend the far-IR study of DOGs to a complete and statistically meaningful sample in order to accurately characterize their far-IR emission and calculate IR luminosities. We generate our DOG catalog using Subaru $r^{+}$ band and MIPS $24 \mu \mathrm{m}$ data from the Cosmological Evolution Survey (COSMOS; Scoville et al. 2007) and combine it with multi-wavelength data in the far-IR from the Herschel Multitiered Extragalactic Survey ${ }^{23}$ (HerMES; Oliver et al. 2012). We

\footnotetext{
22 Herschel is an ESA space observatory with science instruments provided by the European-led Principal Investigator consortia and with important participation from NASA.

23 http://hermes.sussex.ac.uk/
}

calculate IR luminosities, SFRs, and dust temperatures for all DOGs detected at $250 \mu \mathrm{m}$ and employ a stacking analysis to calculate the average properties of the undetected population in the Spectral and Photometric Imaging Receiver (SPIRE) and thus to probe fainter luminosities. For DOGs at $z=1.5-2.5$, we generate a luminosity function and calculate the SFR density at $z \sim 2$.

This paper is organized as follows. Section 2 describes the data set and sample selection. The results and our analysis are presented in Section 3. We summarize our conclusions in Section 4. Unless specifically stated, all magnitudes are reported in the $\mathrm{AB}$ system, where $-2.5 \log _{10} S_{v}(\mu \mathrm{Jy})+23.9=$ $\mathrm{AB}$ mag, and assume a standard $\Lambda \mathrm{CDM}$ cosmology with $H_{0}=$ $70 \mathrm{~km} \mathrm{~s}^{-1} \mathrm{Mpc}^{-1}, \Omega_{\mathrm{M}}=0.3$, and $\Omega_{\Lambda}=0.7$.

\section{DATA AND SAMPLE SELECTION}

\subsection{Far-infrared Data}

The 250, 350, and $500 \mu \mathrm{m}$ far-IR data were obtained using the Herschel/SPIRE (Griffin et al. 2010; Swinyard et al. 2010) as part of HerMES, with an area coverage that completely overlaps with the MIPS observations of the $2 \mathrm{deg}^{2}$ COSMOS field. We use the first data release (DR1) of HerMES maps that were processed using the smap pipeline (Levenson et al. 2010). The reduced maps reach $3 \sigma$ point-source depths of 8,10 , and $14 \mathrm{mJy}$, in the 250,350, and $500 \mu \mathrm{m}$ channels, respectively, where $\sigma$ is the combined instrumental and confusion noise. For sources with $S_{250} \geqslant 3 \sigma$, we use the measured photometry from the HerMES cross-identification catalog. This catalog uses known positions of $24 \mu \mathrm{m}$ sources as a prior, and estimates SPIRE fluxes via linear inversion methods. Model selections are used to account for and prevent overfitting, and to optimize the $24 \mu \mathrm{m}$ input. The fitting method is outlined in more detail in Roseboom et al. (2010).

\subsection{Optical and Mid-infrared Data}

We use deep Subaru Suprime-Cam (Komiyama et al. 2003) aperture-corrected $r^{+}$photometry supplied by the COSMOS catalog (Capak et al. 2007). The $5 \sigma$ point-source depth for a $3^{\prime \prime}$ aperture is $26.8 \mathrm{mag}$.

The near-IR data are from Spitzer observations carried out by the COSMOS Spitzer Survey (S-COSMOS; Sanders et al. 2007) using the Infrared Array Camera (IRAC; Fazio et al. 2004) and MIPS. The IRAC 5 $\sigma$ depths at 3.6, 4.5, 5.6, and $8.0 \mu \mathrm{m}$ for an aperture-corrected $1^{\prime \prime} .9$ aperture, are $0.50,0.6,3$, and $5 \mu \mathrm{Jy}$, respectively. The MIPS $24.0 \mu \mathrm{m} 5 \sigma$ point-source depth is $80 \mu \mathrm{Jy}$ (Le Floc'h et al. 2009).

We next generate a MIPS $24 \mu \mathrm{m}$-selected catalog that combines the Subaru and Spitzer data sets, using a two-step crossmatching process within the $2 \mathrm{deg}^{2}$ of the Subaru deep area in order to find optical counterparts for each source (Fu et al. 2010). Firstly, the $24 \mu \mathrm{m}$ coordinates are matched to the closest IRAC detection within a $2^{\prime \prime}$ search radius, then the nearest optical counterpart is identified within $1^{\prime \prime}$ of the IRAC position. Finally, sources near bright stars that were within the Subaru/ optical and Spitzer/IRAC $3.6 \mu \mathrm{m}$ coverage were removed from the catalog to avoid contamination. The final $24 \mu \mathrm{m}$ catalog is $\geqslant 90 \%$ complete above $S_{24} \geqslant 80 \mu \mathrm{Jy}$ and contains 28,639 sources with $S_{24} \geqslant 100 \mu \mathrm{Jy}$.

\subsection{Sample Selection}

DOGs are selected in the standard manner, by identifying sources with $r^{+}-[24] \geqslant 7.5\left(\mathrm{AB}\right.$ mag; $\left.S_{24} / S_{r^{+}} \geqslant 1000\right)$ 


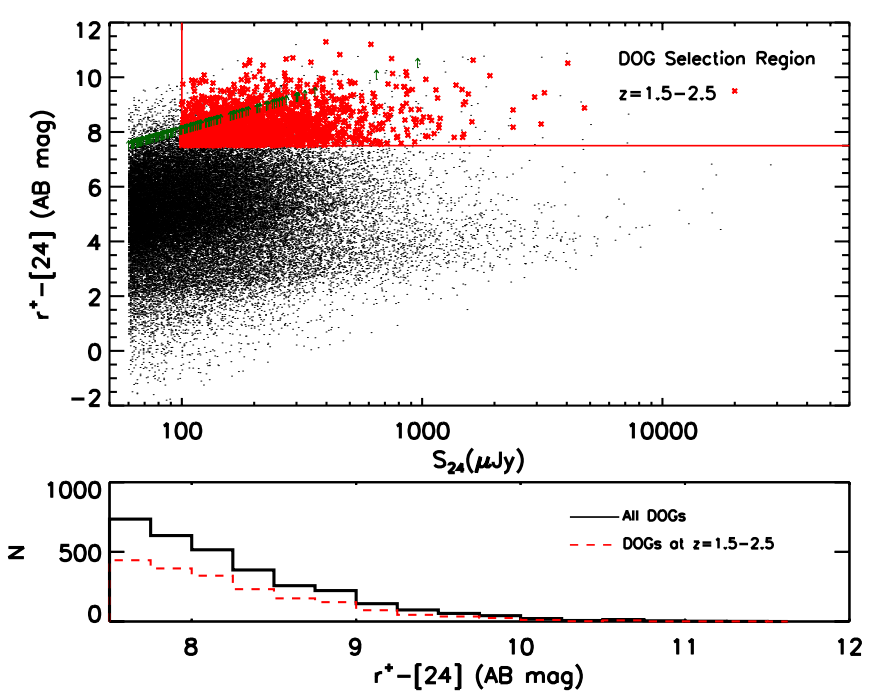

Figure 1. $r^{+}-[24]$ as a function of $24 \mu \mathrm{m}$ flux (top panel) and $r^{+}-[24]$ distribution for DOGs (bottom panel). DOGs are selected to have $r^{+}-[24] \geqslant$ $7.5 \mathrm{AB}$ mag and $S_{24} \geqslant 100 \mu \mathrm{Jy}$. DOGs with $z=1.5-2.5$ are highlighted in red, while green arrows are lower limits for sources that were undetected in the $r^{+}$band. The distribution of $r^{+}-[24]$ for all DOGs compared to those at $z=1.5-2.5$ shows that DOGs in this redshift range are not biased with respect to the full sample.

(A color version of this figure is available in the online journal.)

and we require [24] $\leqslant 18.90 \mathrm{mag}\left(S_{24} \geqslant 100 \mu \mathrm{Jy}\right)$ due to the depth of the $24 \mu \mathrm{m}$ data. Using these criteria, 3077 of the $28,639(11 \%)$ COSMOS $24 \mu \mathrm{m}$ sources with $S_{24} \geqslant 100 \mu \mathrm{Jy}$ are identified as DOGs (Figure 1). The near-IR SED of each DOG is examined using IRAC photometry $(\geqslant 5 \sigma)$ to classify whether a DOG contains a bump-like feature or resembles a power law. For this study, a "bump" DOG is defined as satisfying one of the following: $S_{3.6} \leqslant S_{4.5} \geqslant S_{8.0} ; S_{4.5} \leqslant S_{5.8} \geqslant S_{8.0}$; or $S_{3.6} \leqslant S_{4.5} \geqslant S_{5.8}$. Here $S_{[3.6,4.5,5.8,8.0]}$ represent the flux densities in the four IRAC channels. Conversely, we label a DOG as "power-law" if it satisfies $S_{3.6} \leqslant S_{4.5} \leqslant S_{5.8} \leqslant S_{8.0}$. Previous studies have interpreted sources that feature a bump in the nearIR SED to be the stellar continuum peak at rest-frame $1.6 \mu \mathrm{m}$, tracing stellar emission, and likely star formation dominated (e.g., Yan et al. 2005; Sajina et al. 2007), while a power law is dominated by AGN continuum emission (e.g., Weedman et al. 2006; Donley et al. 2007). Bump DOGs compose $47 \%$ of our sample, while power-law DOGs are rarer, totaling $10 \%$. The remaining $43 \%$ are not classified due to one of two possibilities: insufficient or low signal-to-noise IRAC data or an SED shape that does not satisfy the above criteria. For the latter case, most of the sources are at $z<2$ (median of $z=1.1$ ), such that the rest-frame $1.6 \mu \mathrm{m}$ stellar continuum peak lies outside the wavelength range of the IRAC channels.

\subsection{Redshifts}

All redshifts used in this paper are from COSMOS. Spectroscopic redshifts are used when available (35 sources, 1\%, Lilly et al. 2007; J. Kartaltepe et al., in preparation), although virtually all of our DOG sample (2979 sources, 97\%) use photometric redshifts. The photometric redshifts are derived from 30 photometric bands (Ilbert et al. 2009), providing $\sigma_{\Delta z /(1+z)}=0.02$, for $24 \mu \mathrm{m}$ sources that lie at $z=1.5-3$ and have the same $r^{+}$ mag range as DOGs. The 61 DOGs that are X-ray detected use photometric redshifts that also account for AGN flux variability (Salvato et al. 2009). Also, two sources do not have a redshift

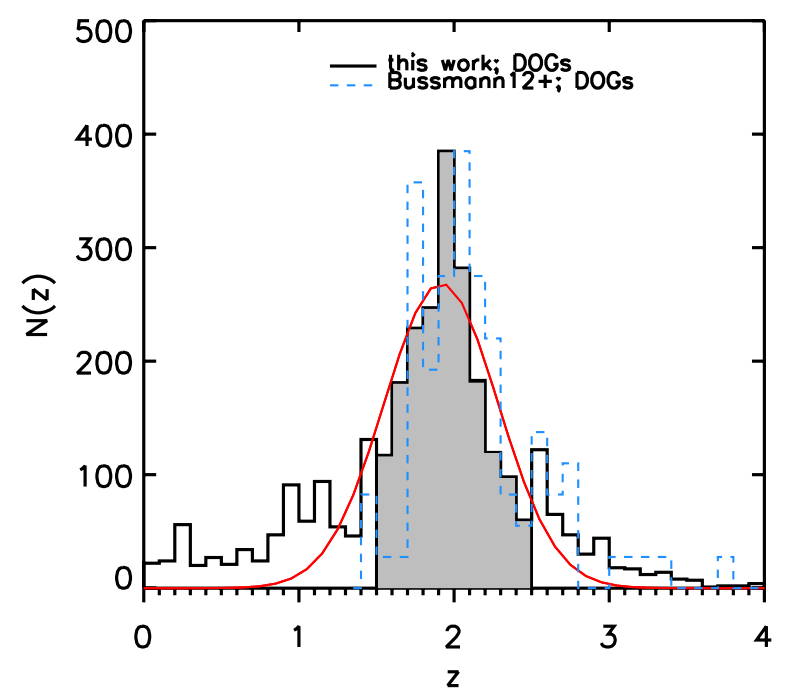

Figure 2. Photometric redshift distribution of DOGs in the COSMOS field. We show the DOG distribution from Bussmann et al. (2012) normalized to have equal peaks for comparison. The filled region highlights the range $z=1.5-2.5$, considered for our analyses in Sections 3.2 and 3.3. We find $\langle z\rangle=1.9 \pm 0.3$, assuming a Gaussian distribution, as shown in red.

(A color version of this figure is available in the online journal.)

estimate and are excluded from our sample. We note that the sharp peak in the redshift distribution at $z=1.95$ is due to rounding from the redshift values associated with the bin size used and no spatial correlation is observed.

The redshift distribution of the final sample of 3075 DOGs is shown in Figure 2, with a mean of $\langle z\rangle=1.9 \pm 0.3$. The sample of 90 DOGs in the Boötes field with spectroscopic redshifts from Bussmann et al. (2012), normalized to have an equal peak with our sample, is also shown. The two samples have a consistent mean $z$ of $1.9 \pm 0.02$ and $2.1 \pm 0.5$, for our sample and the Bussmann et al. (2012) sample, respectively.

\section{ANALYSES AND RESULTS}

\subsection{Far-infrared Spectral Energy Distributions}

Using the COSMOS redshifts and Herschel 250, 350, and $500 \mu \mathrm{m}$ photometry, we fit the far-IR SED and calculate the rest-frame IR luminosity $(8-1000 \mu \mathrm{m})$ and characteristic dust temperature. We divide the DOGs into two subsamples based on $250 \mu \mathrm{m}$ detections because this SPIRE channel offers the deepest far-IR observations and the smallest beam size. A DOG is considered to be Herschel-detected if it satisfies $S_{250} \geqslant 3 \sigma_{250}$ (where $\sigma_{250}$ is the total uncertainty due to the instrumental and confusion noise), and undetected otherwise. Of our DOG sample, $51 \%$ are thus Herschel-detected. To calculate the characteristic dust temperature, for each of these we use the available SPIRE flux densities to fit a modified blackbody of the form

$$
S_{v} \propto B_{v}\left(T_{\text {dust }}\right) v^{\beta}
$$

where $v$ is frequency, $\beta$ is the dust emissivity, fixed to the typical value of 1.5 (Draine 2003), $T_{\text {dust }}$ is the dust temperature and $B_{v}$ is the Planck function, defined as

$$
B_{v}=\frac{2 h v^{3}}{c^{2}} \frac{1}{e^{h v / k_{\mathrm{B}} T_{\mathrm{dust}}}-1}
$$

Here $h$ is Planck's constant, $c$ is the speed of light, and $k_{\mathrm{B}}$ is Boltzmann's constant. The temperature we calculate is insensitive to and consistent with the reported error bars from varying 
Table 1

SPIRE Stacking Results

\begin{tabular}{lccccccc}
\hline \hline $\operatorname{Bin}(n)^{\mathrm{a}}$ & $z$ & $\begin{array}{c}S_{250} \mathrm{~b} \\
(\mathrm{mJy})\end{array}$ & $\begin{array}{c}S_{350} \mathrm{~b} \\
(\mathrm{mJy})\end{array}$ & $\begin{array}{c}S_{500} \mathrm{~b} \\
(\mathrm{mJy})\end{array}$ & $N^{\mathrm{c}}$ & $\begin{array}{c}L_{\mathrm{IR}}{ }^{\mathrm{d}} \\
\left(\times 10^{12} L_{\odot}\right)\end{array}$ & $\begin{array}{c}T_{\text {dust }}{ }^{\mathrm{d}} \\
(\mathrm{K})\end{array}$ \\
\hline 1 & $<1.5$ & $4.2 \pm 0.5$ & $3.0 \pm 0.4$ & $2.1 \pm 0.4$ & 354 & $0.16 \pm 0.13$ & $25.1 \pm 5.5$ \\
2 & $1.50-1.75$ & $3.5 \pm 0.5$ & $2.6 \pm 0.5$ & $1.7 \pm 0.4$ & 218 & $0.37 \pm 0.02$ & $34.6 \pm 0.9$ \\
3 & $1.75-2.00$ & $3.4 \pm 0.5$ & $2.9 \pm 0.4$ & $1.6 \pm 0.4$ & 406 & $0.52 \pm 0.07$ & $37.2 \pm 0.9$ \\
4 & $2.00-2.25$ & $4.3 \pm 0.6$ & $3.9 \pm 0.6$ & $1.7 \pm 0.5$ & 237 & $0.68 \pm 0.06$ & $40.0 \pm 1.0$ \\
5 & $2.25-2.50$ & $4.3 \pm 1.1$ & $3.7 \pm 0.9$ & $2.7 \pm 0.7$ & 82 & $1.00 \pm 0.21$ & $37.8 \pm 0.8$ \\
6 & $>2.5$ & $4.9 \pm 0.7$ & $4.0 \pm 0.6$ & $2.9 \pm 0.6$ & 185 & $1.71 \pm 2.8$ & $44.3 \pm 6.3$ \\
\hline
\end{tabular}

Notes.

${ }^{a}$ Bin number used to label stacked undetected DOGs in Figure 6.

b Measured flux densities are from median stacking. The errors are from bootstrapping.

${ }^{c}$ Number of sources per redshift bin.

$\mathrm{d}$ Average and standard deviation per bin.

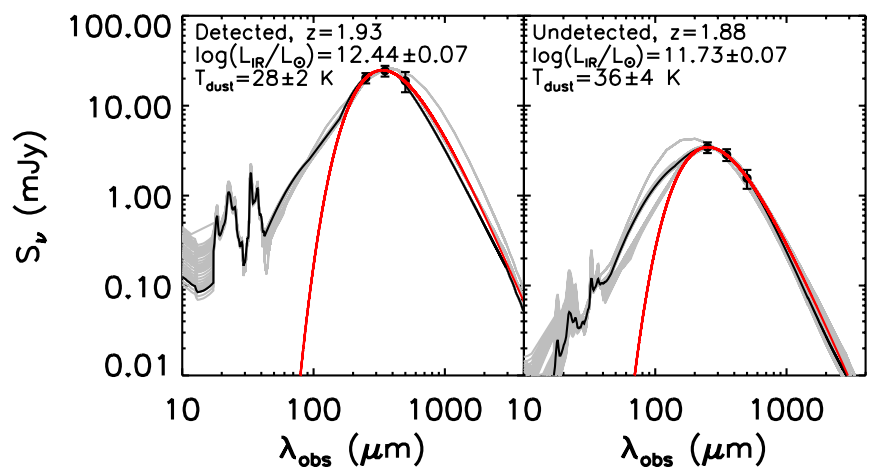

Figure 3. Example SED fitting for a Herschel-detected DOG (left panel) and an undetected DOG (right panel). The black curve shows the best fitting template to the SPIRE data points (black circles) and the gray curves show CE01 templates that provide acceptable fits consistent with the error bars. The red curve shows the best-fit modified blackbody, which we use to calculate the dust temperature. (A color version of this figure is available in the online journal.)

$\beta$ slightly. All $250 \mu \mathrm{m}$ detected sources have measured flux densities at either $350 \mu \mathrm{m}$ or $500 \mu \mathrm{m}$, although it is not required to satisfy the $3 \sigma$ limit (including confusion) in these wavelengths. For the sources that have low significance detections $(\leqslant 3 \sigma)$ at $350 \mu \mathrm{m}$ and/or $500 \mu \mathrm{m}$, we allow the full range of the uncertainties in flux densities when fitting for their IR luminosities and dust temperatures.

We derive estimates of the IR luminosity by fitting the available SPIRE data to the SED template library of Chary \& Elbaz (2001, hereafter CE01). The template with the minimum $\chi^{2}$ is chosen for the best fit. The uncertainty in IR luminosity is derived by first producing 1000 mock catalogs for each source that assume a Gaussian distribution centered around the measured SPIRE flux density, with a dispersion equal to the average flux density error. The IR luminosity per source is recalculated 1000 times and the standard deviation of the IR luminosity distribution is the error in our calculation. Examples of the SED template and modified blackbody fitting are shown in Figure 3.

The IR luminosity $(8-1000 \mu \mathrm{m})$ is converted to SFR using (Kennicutt 1998)

$$
\operatorname{SFR}\left(M_{\odot} \mathrm{yr}^{-1}\right)=1.72 \times 10^{-10} L_{\mathrm{IR}}\left(L_{\odot}\right),
$$

which assumes a Salpeter initial mass function (IMF). We note that in our study we assume that UV emission will provide a negligible contribution to the total SFR, as validated by Penner et al. (2012).
To measure the average flux density of the Herschelundetected DOGs, we bin the sources in redshift and for each bin stack on the SPIRE residual maps. These maps are generated by performing a blind extraction and point-spread function subtraction to prevent contamination of individually detected sources. We use the publicly available IDL stacking library from Béthermin et al. (2010) to perform the stacking. ${ }^{24}$ Each stacked image is converted from the native $\mathrm{Jy} \mathrm{beam}^{-1}$ to $\mathrm{Jy} \mathrm{pixel}^{-1}$ and aperture photometry with an aperture size equal to $22^{\prime \prime}, 30^{\prime \prime}$, and $42^{\prime \prime}$ for 250,350 , and $500 \mu \mathrm{m}$, respectively, is performed to calculate the flux of the stacked images. These aperture flux densities are consistent with those measured in the central pixel when the stacked map is in units of $\mathrm{Jy}_{\text {beam }}{ }^{-1}$.

The observed stacked flux densities are corrected for the boosting from clustering bias by dividing by factors of 1.07, 1.10 , and 1.20 at 250, 350, and $500 \mu \mathrm{m}$, respectively. The appropriate correction factors vary with clustering strength and are thus population dependent. These values were calculated by Béthermin et al. (2012) for $24 \mu \mathrm{m}$ sources and are valid for DOGs because the observed correlation lengths, $r_{0}$ (a proxy for clustering amplitude), for DOGs (Brodwin et al. 2008) and the parent population of $24 \mu \mathrm{m}$ sources (Magliocchetti et al. 2008; Starikova et al. 2012) are consistent. Errors in the photometry are calculated from bootstrapping the sources to be stacked. For each redshift bin, the clustering-corrected SPIRE flux densities of Herschel-undetected DOGs are set to equal the median stacked flux densities and the IR luminosity and dust temperature are calculated using the same method as for the Herschel-detected DOGs. The (clustering-corrected) stacked fluxes and errors, and the resulting average IR luminosities and dust temperatures, are shown in Table 1.

We note that the average stacked $250 \mu \mathrm{m}$ flux density for the Herschel-undetected DOGs is $4.1 \pm 0.7 \mathrm{mJy}$, which is a factor of two lower than the $250 \mu \mathrm{m}$ catalog detection limit. In Figure 4 we show an example of the median stacked images for 250,350 , and $500 \mu \mathrm{m}$ from left to right at $z=1.75-2.00$ and an example SED using stacked SPIRE flux densities for a Herschel-undetected DOG at $z=1.88$ is shown in the right panel of Figure 3. Each image stack is large enough to provide a good estimate for the background noise.

Figure 5 shows IR luminosities of the Herschel-detected DOGs as a function of redshift. The average IR luminosity for Herschel-detected and undetected DOGs is $(2.8 \pm 0.3) \times 10^{12} L_{\odot}$ and $(6.0 \pm 1.0) \times 10^{11} L_{\odot}$, respectively. LIRGs $\left(10^{11} \leqslant\right.$

\footnotetext{
24 The IDL stacking library from Béthermin et al. (2010) is available at http://www.ias.u-psud.fr/irgalaxies/downloads.php.
} 

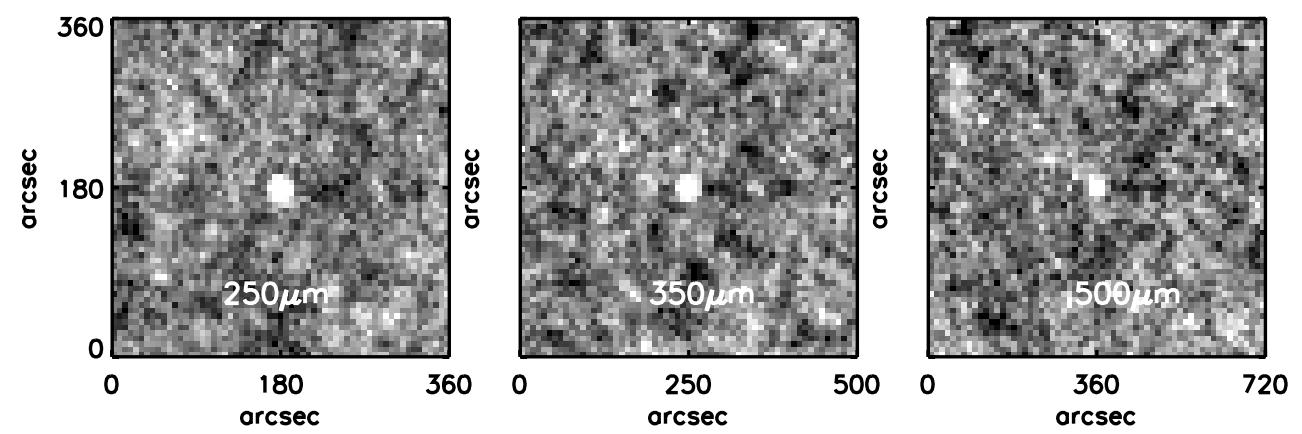

Figure 4. Example median stacking results of Herschel-undetected DOGs at 250, 350, and $500 \mu \mathrm{m}$ for undetected DOGs at $z=1.75-2.00$.

Table 2

Average DOG IR Luminosities and Dust Temperatures

\begin{tabular}{lcc}
\hline \hline Type & $\begin{array}{c}L_{\mathrm{IR}} \\
\left(\times 10^{12} L_{\odot}\right)\end{array}$ & $\begin{array}{c}T_{\text {dust }} \\
(\mathrm{K})\end{array}$ \\
\hline Detected $^{\mathrm{a}}$ & $2.8 \pm 0.3$ & $33 \pm 7$ \\
Undetected $^{\mathrm{a}}$ & $0.6 \pm 0.1$ & $37 \pm 5$ \\
Bump $^{\mathrm{b}}$ & $4.5 \pm 0.4$ & $34 \pm 7$ \\
Power-law $^{\mathrm{b}}$ & $3.1 \pm 0.4$ & $37 \pm 6$ \\
\hline
\end{tabular}

Notes.

a Detected sources satisfy: $S_{250} \geqslant 3 \sigma$; undetected sources have $S_{250}<3 \sigma$.

b Power-law (AGN-dominated) and bump (star-forming) median IR luminosities are from the detected sample.

$\left.L_{\mathrm{IR}}\left(L_{\odot}\right) \leqslant 10^{12}\right)$ comprise $15 \%$ of Herschel-detected DOGs and $75 \%$ of Herschel-undetected DOGs. ULIRGs $\left(10^{12} \leqslant\right.$ $\left.L_{\mathrm{IR}}\left(L_{\odot}\right) \leqslant 10^{13}\right)$ make up $78 \%$ of the Herschel-detected and $15 \%$ of the Herschel-undetected DOGs. Hyper-luminous infrared galaxies (HLIRGs; $\geqslant 10^{13} L_{\odot}$ ) are the rarest, totaling $2 \%$ of the Herschel-detected DOGs and none of the Herschelundetected DOGs. Although we note that there is additional uncertainty in the fractional contributions of the undetected sources, due to the use of stacked average fluxes, which minimizes the contribution from extreme sources. Herscheldetected power-law, or AGN-dominated, DOGs have on average $L_{\mathrm{IR}}=(4.5 \pm 0.5) \times 10^{12} L_{\odot}$, making them more IR-luminous than Herschel-detected bump, star-forming DOGs, which have $L_{\mathrm{IR}}=(3.1 \pm 0.4) \times 10^{12} L_{\odot}$, which is consistent with the findings of Melbourne et al. (2012). The results for the average IR luminosities and dust temperatures of the different subsamples of DOGs considered for this study are summarized in Table 2. Selection effects are investigated by calculating the IR luminosity of a representative CE01 template, scaled such that $S_{24}=100 \mu \mathrm{Jy}$ or $S_{250}=8 \mathrm{mJy}$, as shown in Figure 5 . The local maxima in IR luminosity at $z \sim 1.5$ in the $24 \mu \mathrm{m}$ curve is due to the rest-frame $9.7 \mu \mathrm{m}$ silicate absorption feature. The lack of DOGs below the $24 \mu \mathrm{m}$ and $250 \mu \mathrm{m}$ limit at a given redshift leads us to conclude that the apparent trend in Figure 5 that IR luminosity is increasing with redshift is a selection effect.

Figure 6 shows dust temperature as a function of IR luminosity for DOGs, color-coded by redshift. The average characteristic dust temperature is $T_{\text {dust }}=(34 \pm 7) \mathrm{K}$ and $(37 \pm 5) \mathrm{K}$ for Herschel-detected and undetected DOGs, respectively. Herschel-detected power-law DOGs and bump DOGs have average $T_{\text {dust }}=(37 \pm 6) \mathrm{K}$ and $(35 \pm 7) \mathrm{K}$, respectively, which is consistent with each other. We investigate sample selection effects in the $T_{\text {dust }}-L_{\text {IR }}$ plane by considering both the IR luminosity of fixed temperature modified blackbody SEDs (Equation (1)) and the CE01 templates at $z=0.5$ and $z=2.0$

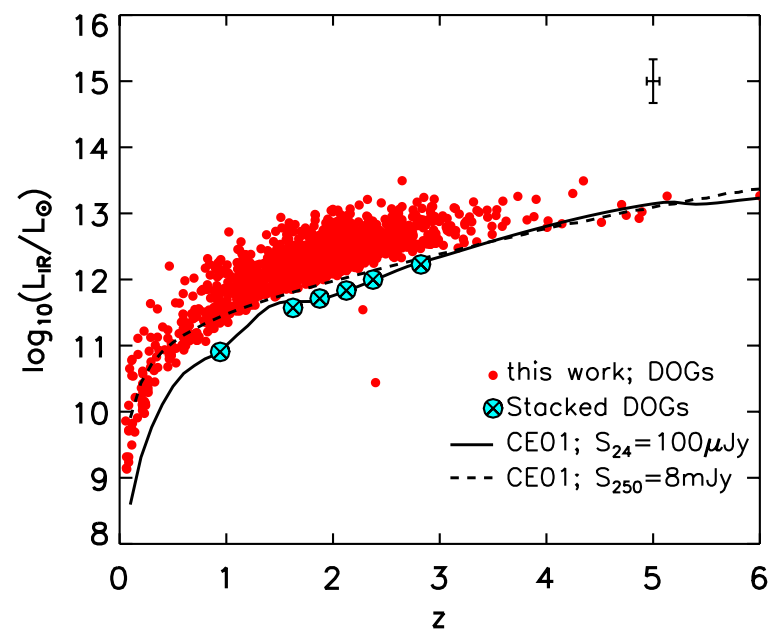

Figure 5. IR luminosity as a function of redshift for Herschel-detected DOGs and the median IR luminosity for stacked DOGs. A representative template from Chary \& Elbaz (2001) scaled to the DOG $24 \mu \mathrm{m}$ (solid curve) and $250 \mu \mathrm{m}$ detection limit (dashed curve) are also shown. A typical error bar is shown at the top right. The apparent trend that IR luminosity increases with redshift is a selection effect.

(A color version of this figure is available in the online journal.)

for $S_{250}=8 \mathrm{mJy}$ (Figure 6). The temperatures of the CE01 SEDs are calculated by fitting the template 250,350 , and $500 \mu \mathrm{m}$ flux densities with a modified blackbody as in our data. Figure 6 also confirms that the $250 \mu \mathrm{m}$ flux density limit biases against lower luminosity sources and the luminosity limit is a function of redshift (see also Figure 5). Furthermore, the apparent correlation between $T_{\text {dust }}$ and $z$ is in fact caused by a combination of the redshift-dependent $L_{\mathrm{IR}}$ selection limit and the correlation between $L_{\mathrm{IR}}$ and $T_{\text {dust }}$ (e.g., Symeonidis et al. 2013; Hwang et al. 2010). The selection limits at $z=0.5$ on Figure 6 also show that at low redshift $(z \lesssim 1)$ there is a bias against the warmer sources, which results in an apparent difference in the dust temperature distribution of Herschel-detected DOGs compared to the observed relationship locally and at higher redshifts (e.g., Chapman et al. 2004; Symeonidis et al. 2013; Hwang et al. 2010). The $24 \mu \mathrm{m}$ flux density limit produces a similar effect as the $250 \mu \mathrm{m}$ limit.

The mean stacked IR luminosities and dust temperatures, per redshift bin, of the Herschel-undetected DOG population are displayed in Figure 6 and are less sensitive to these selection biases. The lowest redshift bin (bin $1, z \leqslant 1.5$ ) is offset relative to the other redshift bins because it covers a wide redshift interval in which the IR luminosity limit has a steep slope (Figure 5). The dearth of sources at high luminosities and low dust temperatures is not a selection effect as these sources would have been detected by our data. This is consistent with 


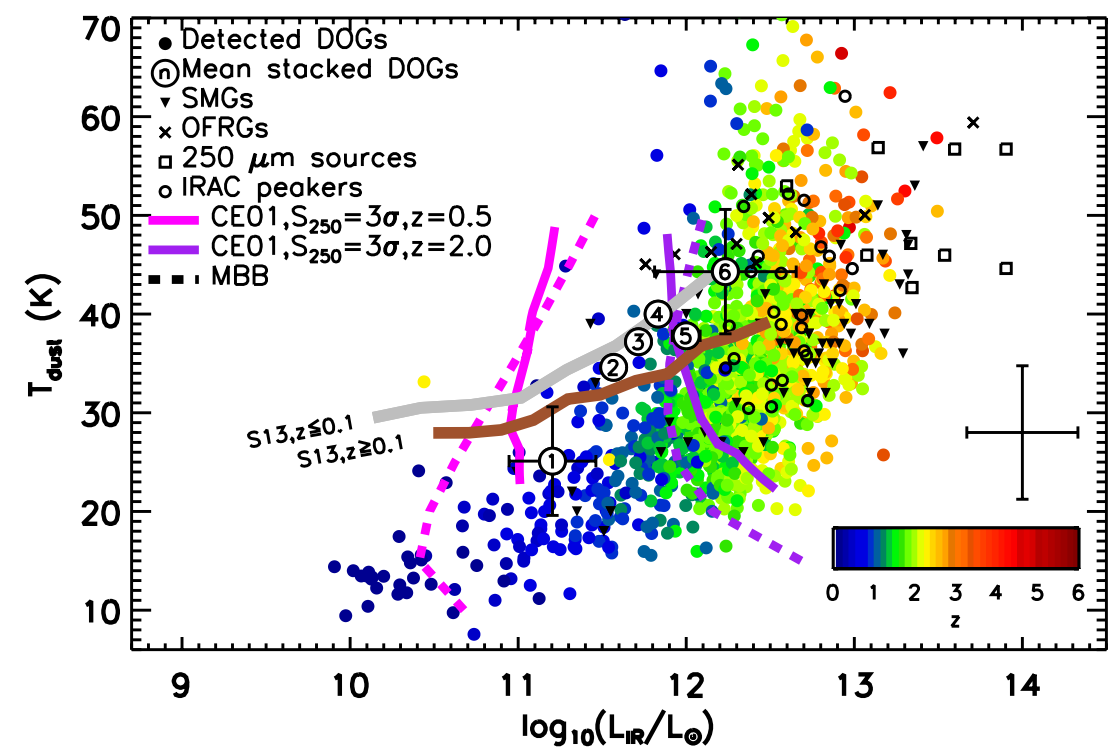

Figure 6. Dust temperatures and infrared luminosities for DOGs compared to other $z \sim 2$ galaxy populations (SMGs, Magnelli et al. 2012; OFRGs, Casey et al. 2009; Magnelli et al. 2010; $250 \mu \mathrm{m}$ sources, Casey et al. 2011; IRAC peakers, Magdis et al. 2010). We show Herschel-detected DOGs, colored by redshift. The mean dust temperature and IR luminosity per redshift bin for the stacked DOGs are labeled by their bin number from Table 1, with their dispersions shown as error bars. We note that some of the bins have dispersions that are too small to display and thus appear to be invisible. The magenta and purple solid curves are generated from estimating the dust temperature and calculating IR luminosity from Chary \& Elbaz (2001) templates at $z=0.5$ and $z=2.0$, respectively, with $S_{250}=8$ mJy, our $3 \sigma$ detection limit. The dashed curves are generated from calculating the IR luminosities of Equation (1) at fixed temperatures, also analyzed at $z=0.5$ and $z=2$. The gray and brown curves are the $T_{\mathrm{dust}}-L_{\mathrm{IR}}$ relations for $z \leqslant 0.1$ and $z \geqslant 0.1$, respectively, from Symeonidis et al. (2013). We conclude that the apparent trend that hotter sources are at higher redshifts is caused by the $T_{\text {dust }}-L_{\mathrm{IR}}$ relation and the redshift-dependent selection limit in $L_{\mathrm{IR}}$. The absence of warm, low luminosity (low redshift) sources is a selection effect. However, cool, high luminosity sources would be detected in our data, and the dearth of these sources is not a selection effect.

(A color version of this figure is available in the online journal.)

Symeonidis et al. (2013), who found that cold cirrus-dominated SEDs (Rowan-Robinson et al. 2010) are rare in the most IR luminous galaxies.

The results shown in Figure 6 suggest that $z \sim 2$ DOGs span a wider range of dust temperatures than $z \sim 2$ SMGs (by which we mean $850 \mu \mathrm{m}$ or $1 \mathrm{~mm}$ selected sources) due to the different selection effects associated with each galaxy population. SMGs are biased toward detecting cold-dust dominated sources $\left(T_{\text {dust }} \sim 30-40 \mathrm{~K}\right)$ because hot sources are missed by submillimeter surveys (Chapman et al. 2004; Casey et al. 2009; Chapin et al. 2009). The discovery of optically faint radio galaxies (OFRGs; Chapman et al. 2004; Casey et al. 2009; Magnelli et al. 2010) at $z \sim 2$, which have similar stellar masses, radio luminosities, and UV spectra as SMGs, but have $T_{\text {dust }} \sim 40-60 \mathrm{~K}$, demonstrate this, while we also note that the radio-detection limit is biased against the coldest sources (e.g., Wardlow et al. 2011). DOGs are more insensitive to these selection biases and thus show a wider range of temperatures at $z \sim 2$. Magdis et al. (2010) found similar results when investigating the characteristic dust temperatures for IRAC peakers and showed that mid-IR selected sources bridge the gap in temperature ranges between OFRGs and SMGs. We note that the $250 \mu \mathrm{m}$ selected sources suffer from the same selection biases as our Herschel-detected DOGs but shifted to higher luminosities due to their shallower $250 \mu \mathrm{m}$ detection limit.

\subsection{Infrared Luminosity Function at $z \sim 2$}

We compute the IR luminosity function of DOGs using the $1 / V_{\max }$ method (Schmidt 1978), defined as

$$
\Phi(L) \Delta L=\sum_{i} \frac{1}{V_{\max , i}},
$$

Table 3

The IR Luminosity Function for DOGs at $z=1.5-2.5$

\begin{tabular}{lcc}
\hline \hline $\log _{10}\left(L_{\mathrm{IR}} / L_{\odot}\right)$ & $\begin{array}{c}\Phi \\
\left(\mathrm{Mpc}^{-3} \mathrm{dex}^{-1}\right)\end{array}$ & $N^{\mathrm{a}}$ \\
\hline $11.40-11.80$ & $(-3.79 \pm 0.02)^{\mathrm{b}}$ & $(660)^{\mathrm{b}}$ \\
$11.80-12.15$ & $-4.17 \pm 0.21(-3.98 \pm 0.22)^{\mathrm{b}}$ & $150(433)^{\mathrm{b}}$ \\
$12.15-12.50$ & $-4.03 \pm 0.03(-4.02 \pm 0.09)^{\mathrm{b}}$ & 522 \\
$12.50-12.85$ & $-4.49 \pm 0.03$ & 252 \\
$12.85-13.20$ & $-5.40 \pm 0.09$ & 31 \\
\hline
\end{tabular}

Notes.

${ }^{\text {a }} N$ is the number of sources per luminosity bin.

${ }^{\mathrm{b}}$ Numerical values in parenthesis include the stacked contribution.

where $V_{\max }$ is the maximum comoving volume of the $i$ th source such that it would be detected and included in the sample. We consider the peak of the redshift distribution using only DOGs at $z=1.5-2.5$. For the Herschel-detected DOGs we use two flux limits to determine $V_{\max }: S_{24}=100 \mu \mathrm{Jy}$ and $S_{250}=8 \mathrm{mJy}$. These are the two detection limits of the survey. For the Herschel-undetected sample, the $24 \mu \mathrm{m}$ flux limit alone was used to calculate $V_{\max }$, as all redshift bins are detected in the stacks. We then calculate each Herschel-undetected DOG's IR luminosity and contribution to the luminosity function using its redshift and the relevant stacked flux densities. The uncertainties are from Poisson statistics and binning errors, where the binning errors are calculated by generating the IR luminosity function 1000 times from IR luminosities calculated from artificial SPIRE flux densities described in Section 3.1 and taking the standard deviation per IR luminosity bin. The DOG IR luminosity function at $z \sim 2$ is presented in Figure 7 and Table 3. The faint end of the IR luminosity function for Herscheldetected and undetected DOGs is coadded, which affects the 


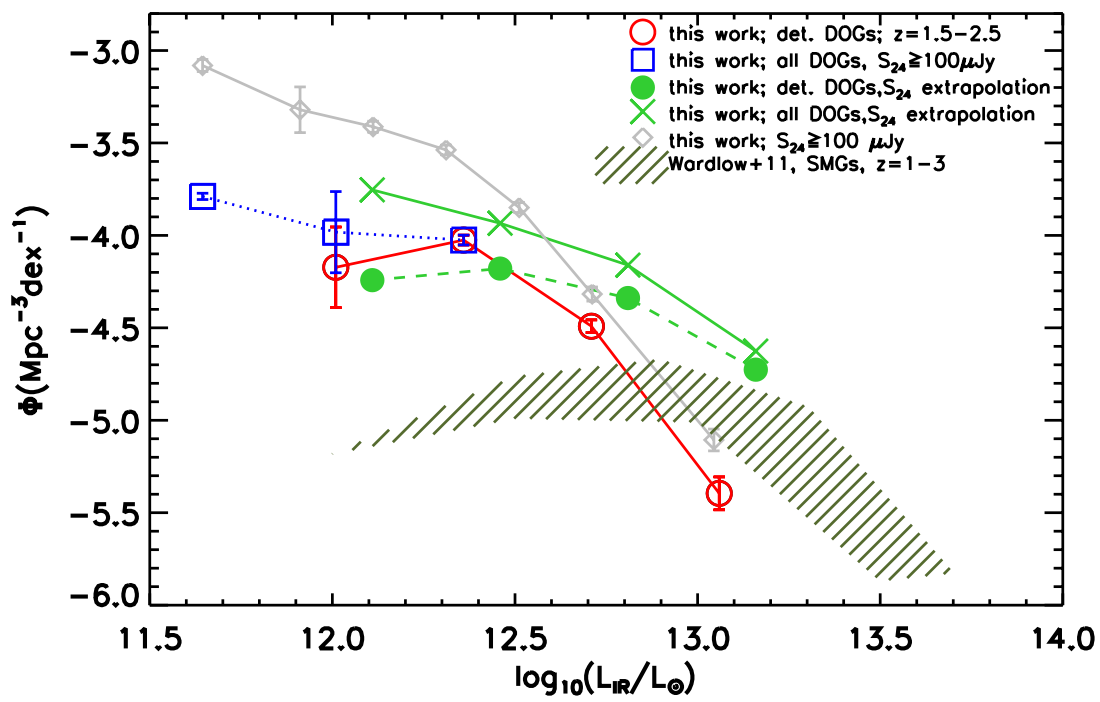

Figure 7. IR luminosity function of DOGs in the COSMOS field at $z=1.5-2.5$ with $S_{24} \geqslant 100 \mu \mathrm{Jy}$. Individually Herschel-detected DOGs and the results from stacking undetected DOGs are shown. We compare this to an IR luminosity function for Herschel-detected DOGs and all DOGs generated from $24 \mu \mathrm{m}$ extrapolation using templates from Chary \& Elbaz (2001), classical SMGs (Wardlow et al. 2011), and $24 \mu \mathrm{m}$ selected galaxies with $S_{24} \geqslant 100 \mu \mathrm{Jy}$. The results of stacking allow us to estimate the faint end of the LF and we note that using $24 \mu \mathrm{m}$ flux density to calculate IR luminosity results in overestimation. DOGs have a higher normalization, $\Phi^{*}$, but a lower luminosity turnover, $L^{*}$, than SMGs.

(A color version of this figure is available in the online journal.)

lowest luminosity bin for Herschel-detected DOGs the most, showing a 0.20 dex increase.

For comparison, the DOG IR luminosity function for Herschel-detected DOGs and all DOGs, calculated by extrapolating the IR luminosity from $S_{24}$ using CE01 templates, is also shown in Figure 7. We find that the IR luminosities using this method are overestimated by a median factor of 1.8 , consistent with the previous studies of $24 \mu \mathrm{m}$ selected galaxies at $z \sim 2$ (Houck et al. 2005; Yan et al. 2007; Daddi et al. 2007; Papovich et al. 2007; Pope et al. 2008; Nordon et al. 2010, 2012; Elbaz et al. 2010, 2011; Magnelli et al. 2011), and affects both the shape and normalization of the IR luminosity function.

We compare the number densities of DOGs to the parent population of sources with $S_{24} \geqslant 100 \mu \mathrm{Jy}$ (Figure 7). The luminosity function of $24 \mu \mathrm{m}$ sources is calculated self-consistently using SPIRE data, including stacking on the Herschel-undetected galaxies. There are 5932 sources in COSMOS with $S_{24} \geqslant$ $100 \mu \mathrm{Jy}$ at $z=1.5-2.5$, of which $32 \%$ are DOGs. Figure 7 shows that DOGs have a smaller overall normalization in their luminosity function (since they are fewer in number) and their relative contribution to the $24 \mu \mathrm{m}$ number density increases with luminosity in agreement with the IR luminosity distribution of DOGs compared to $24 \mu \mathrm{m}$ sources in Penner et al. (2012).

Figure 7 also shows the $z=1-3$ SMG luminosity function by combining the $z=1-2$ and $z=2-3$ redshift bins from Wardlow et al. (2011) for comparison of the distinct, but overlapping DOG and SMG (Pope et al. 2008) populations. DOGs are more common than SMGs at $z \sim 2$, which is reflected in the higher normalization in the luminosity function, although in the HLIRG regime, SMGs dominate. This is consistent with the picture in which DOGs represent an evolutionary stage toward the end of the peak of SFR, observed as the SMG phase (Narayanan et al. 2010), they must have lower IR luminosities and SFRs on average. In this case, the relative scaling of the two luminosity functions indicates that the DOG phase is longer lived than the SMG phase. As is shown in Figure 8, the total star formation rate density $\left(\rho_{\mathrm{SFR}}\right)$ provided by the two populations are approximately even despite the number and intensity of the sources.

To calculate the contribution of DOGs with $S_{24} \geqslant 100 \mu \mathrm{Jy}$ to the $\rho_{\mathrm{SFR}}$ of the universe at $z \sim 2$, we integrate the IR luminosity function and use Equation (3). Figure 8 shows DOGs compared to other $z \sim 2$ galaxy populations. The total uncertainty in $\rho_{\mathrm{SFR}}$ is calculated from the quadrature sum of individual SFR uncertainties and the standard deviation of $\rho_{\text {SFR }}$ from the mock catalogs discussed in Section 3.1. Horizontal error bars represent the considered redshift interval. The value of $\rho_{\mathrm{SFR}}$ for DOGs at $z=1.5-2.5$ is $(3.2 \pm 0.5) \times 10^{-2} M_{\odot} \mathrm{yr}^{-1} \mathrm{Mpc}^{-3}$ which contributes to $12 \%-29 \%$ of the overall $\rho_{\mathrm{SFR}}$ at $z=2$ calculated from UV and IR data shown by Hopkins \& Beacom (2006) and Burgarella et al. (2013). When comparing to $z=$ $1.5-2.5$ sources with $S_{24} \geqslant 100 \mu \mathrm{Jy}$, DOGs contribute $33 \%$ to the $24 \mu \mathrm{m} \rho_{\text {SFR }}$.

The Herschel-undetected and power-law sources provide non-dominant contributions to the total $\rho_{\mathrm{SFR}}$ of DOGs. The Herschel-undetected DOGs contribute $18 \%$ and power-law DOGs contribute just $9 \%$. We note that even though power-law DOGs are thought to be dominated by AGN emission in the IRAC bands, their far-IR emission is still likely dominated by star formation, as is the case for other far-IR luminous samples containing AGNs (Elbaz et al. 2010; Lutz et al. 2008, 2010). Indeed, even studies of the most active AGNs have revealed that SED fits for Herschel-detected AGNs always required a starburst component in order to appear bright in the far-IR (Hatziminaoglou et al. 2010). As an attempt to quantify this claim, we use a simplified method to calculate an upper limit on the AGN contribution to the IR luminosity and SFR in powerlaw DOGs and hence the contamination of $\rho_{\text {SFR }}$ by AGNs. We begin by scaling the AGN SEDs from Kirkpatrick et al. (2012) to the $24 \mu \mathrm{m}$ flux density of each power-law DOG and calculate the luminosity from the warm dust component. Then, by assuming that the warm dust component is entirely AGN-dominated and the cold-dust component is entirely star formation dominated, we can subtract the warm IR luminosity from the CE01 IR luminosity to calculate the residual contribution from star 


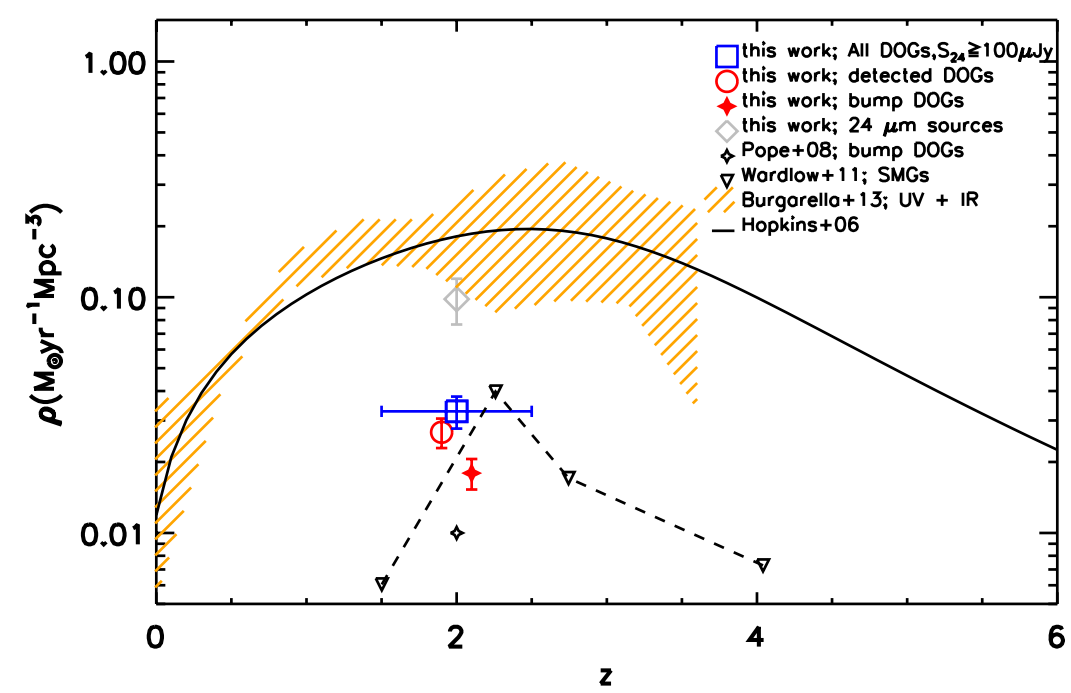

Figure 8. Star formation rate density ( $\left.\rho_{\mathrm{SFR}}\right)$ of all DOGs with $S_{24} \geqslant 100 \mu \mathrm{Jy}$, Herschel-detected DOGs (offset by $z=-0.1$, for clarity), bump DOGs (offset by $z=+0.1$, for clarity) and all $S_{24} \geqslant 100 \mu \mathrm{Jy}$ sources in COSMOS at $z=1.5-2.5$. We also show $\rho_{\mathrm{SFR}}$ for bump DOGs $\left(S_{24} \geqslant 100 \mu \mathrm{Jy}\right)$ at $z=2$ in the GOODS field from Pope et al. (2008) and SMGs from Wardlow et al. (2011). The evolution of $\rho_{\mathrm{SFR}}$ as a function of redshift from Hopkins \& Beacom (2006) and Burgarella et al. (2013) are also shown. Based on these models, DOGs contribute $12 \%-29 \%$ to the total $\rho_{\mathrm{SFR}}$ of the universe at $z \sim 2$.

(A color version of this figure is available in the online journal.)

formation. We find that power-law DOGs each have a maximum average contribution of $70 \%$ to the IR luminosity, which could contaminate $\rho_{\mathrm{SFR}}$ by $\sim 0.2 \times 10^{-2} M_{\odot} \mathrm{yr}^{-1} \mathrm{Mpc}^{-3}$, which is only $6 \%$ of the total DOG $\rho_{\text {SFR }}$. In addition, we also estimate the dispersion of AGN contribution by normalizing quasar SED templates from Elvis et al. (1994), Richards et al. (2006), Polletta et al. (2007), and Dai et al. (2012) to the average power-law DOG $24 \mu \mathrm{m}$ flux density at $z=1.5-2.5$ and assume that the SEDs have no emission associated with star formation. Under this assumption, the average AGN contributions to the individual galaxies' IR luminosities range from $5 \%$ to $65 \%$, depending on the SED, which corresponds to $0.005 \%$ to $6 \%$ contribution to the total DOG $\rho_{\mathrm{SFR}}$.

We note that Pope et al. (2008) also examined bump (starforming) DOGs at $z \sim 2$ down to $S_{24}=100 \mu \mathrm{Jy}$ and they calculated $\rho_{\mathrm{SFR}} \sim 1 \times 10^{-2} M_{\odot} \mathrm{yr}^{-1} \mathrm{Mpc}^{-3}$, under the assumption that the average DOG has an SFR of $200 M_{\odot} \mathrm{yr}^{-1}$. This value is lower than the bump $\rho_{\mathrm{SFR}}=1.9 \pm 0.3 \times$ $10^{-2} M_{\odot} \mathrm{yr}^{-1} \mathrm{Mpc}^{-3}$ that we measure. However, it is difficult to determine whether these two values are significantly different because Pope et al. (2008) do not provide an error on their measurement. We use their reported fractional error on the average IR luminosity $\left((1.1 \pm 0.7) \times 10^{12} L_{\odot}\right)$ to estimate that the minimum error on their $\rho_{\mathrm{SFR}}$ is $\sim 0.6 \times 10^{-2} M_{\odot} \mathrm{yr}^{-1} \mathrm{Mpc}^{-3}$, in addition to the contribution from the counting error from their 62 sources (compared to our 1137 bump sources at $z=1.5-2.5$ ). We also note that the selection criteria for the two studies are slightly different and if we were to use the bump DOG selection scheme in Pope et al. (2008; $S_{3.6}<S_{4.5}>S_{5.8}$ and $S_{4.5}>S_{8.0}$, or $S_{4.5}<S_{5.8}>S_{8.0}$ and $S_{3.6}>S_{8.0}$ ) we would identify 100 fewer bump DOGs (9\% of our sample of bump DOGs are at $z=1.5-2.5$ ). We conclude that the two results are consistent but since our study uses a larger sample and employs a combination of direct observations and redshift-binned stacking to determine our IR luminosities, we consider this measurement more accurate.

\subsection{Stellar Mass Build-up}

Using the stellar masses derived in Ilbert et al. (2010, corrected to assume a Salpeter IMF by adding +0.24 dex and to be consistent with our SFR calculations) and our derived SFRs using Herschel data, we investigate where DOGs lie in the star formation rate-stellar mass (SFR- $M_{*}$ ) plane. Disk galaxies with a steady star formation mode are observed to form a tight correlation in their SFRs as a function of stellar mass, defining a "main sequence" (Daddi et al. 2007; Elbaz et al. 2011). Outliers above this relation are thought to be merger-driven starburst galaxies (Rodighiero et al. 2011 and references therein). In the top panel of Figure 9 we show the SFRs and stellar masses for Herschel-detected DOGs, considering only those at $z=1.5-2.5$ to minimize the effects of redshift evolution. Average error bars are plotted for SFRs and the uncertainties in stellar mass are fixed to $0.5 \mathrm{dex}$, which covers the systematic offset range due to the choice of extinction laws and stellar population synthesis models.

Figure 9 shows that power-law DOGs and bump DOGs cover the same ranges in stellar mass and star formation rate in the $\mathrm{SFR}-M_{*}$ plane, as expected if the far-IR is star formation dominated. Our findings are also consistent with previous studies that investigated the similarities in properties of farIR SEDs of Herschel-selected star-forming galaxies and AGN (Mullaney et al. 2012).

The IR main sequence from Elbaz et al. (2011) for Herschelselected star-forming galaxies at $z=2$ is also shown in Figure 9. DOGs have a significant amount of scatter about this relation, with $46 \%$ within a factor of two of the main sequence, $24 \%$ above it and consistent with starbursts, and $31 \%$ below it in the more quiescent regime.

The bottom panel of Figure 9 shows the specific SFR $\left(\mathrm{sSFR}=\mathrm{SFR} / M_{*}\right)$ as a function of stellar mass. The sSFR quantifies the weighted SFR and stellar mass, with its inverse giving the mass-doubling time for the current episode of star formation activity. An apparent negative correlation in which lower mass $z=2$ DOGs exhibit higher sSFRs than their higher mass counterparts is observed; however, this is largely a selection effect due to the flux limit of our sample. On the top panel of Figure 9 we use the minimum IR luminosity at $z=2$ from our sample to represent a minimum detectable SFR limit, shown as the horizontal line. We convert this to sSFR for a range of masses and this is shown as the diagonal line in the bottom 


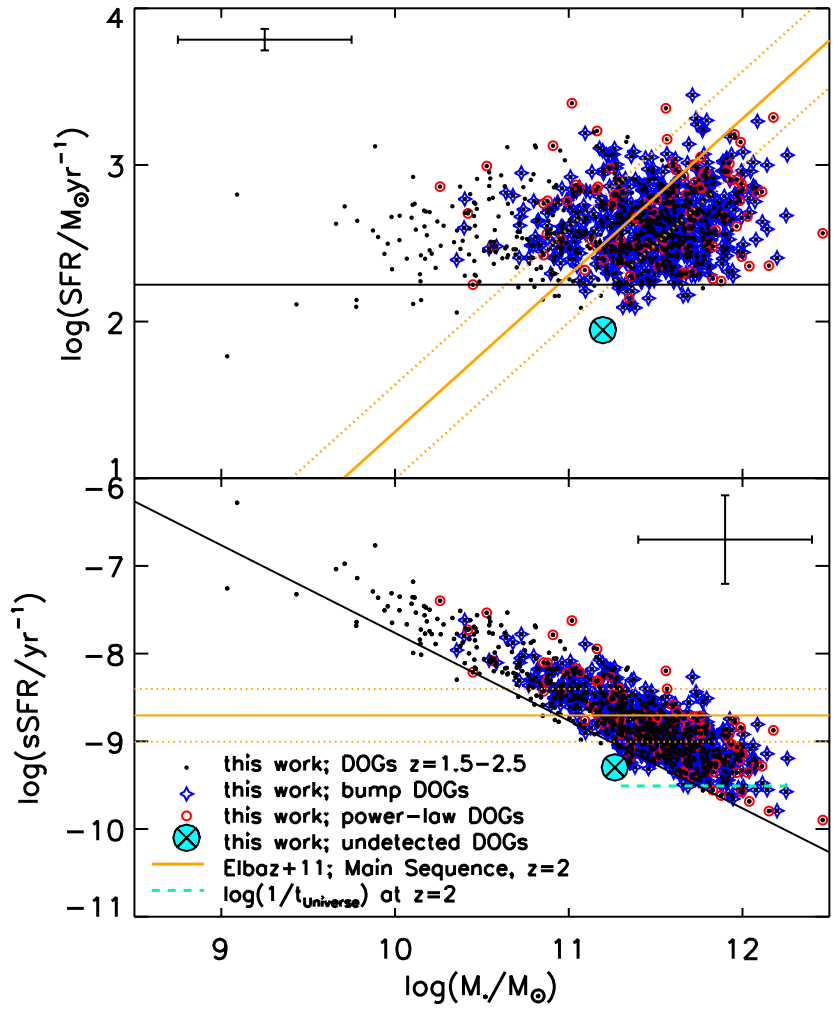

Figure 9. Star formation rate (SFR; top panel) and specific star formation rate (sSFR; bottom panel) as a function of stellar mass for DOGs at $z=1.5-2.5$. Power-law DOGs and bump DOGs are statistically indistinguishable in the SFR- $M_{*}$ plane. The sSFR at $z=2$ using the relation for star-forming galaxies from Elbaz et al. (2011) and its conversion to SFR for the displayed range of masses is shown as the thick solid orange line in both panels. The orange dotted lines represent a factor of two dispersion from the derived SFR and SSFR. DOGs have a large scatter about the main sequence relation, having sources in the starburst, main sequence, and passive galaxy regimes. The thin horizontal black line in the top panel represents a minimum detectable SFR at $z \sim 2$, caused by the $24 \mu \mathrm{m}$ flux density limit. Converting this to an sSFR value results in the diagonal line in the bottom panel, leading us to conclude that the apparent negative correlation between sSFR and stellar mass is a selection effect.

(A color version of this figure is available in the online journal.)

panel. The logarithmic inverse age of the universe in Gyr at $z=2$ is $\approx-9.5$ (dashed line in Figure 9) and most DOGs have sSFRs larger than this, indicating that the observed phase of star formation could be responsible for their total observed stellar mass.

Finally, we use the known redshift distribution and the sSFRs of DOGs to compare their volume densities to their proposed progenitors, SMGs. The volume density of observed DOGs with $S_{24} \geqslant 100 \mu \mathrm{Jy}$ at $z=1.5-2.5$ is $8 \times 10^{-5} \mathrm{Mpc}^{-3}$. Using the median DOG sSFR to estimate the characteristic lifetime of the DOG phase to be approximately $1 \mathrm{Gyr}$, we can correct this density for the burst duty cycle to derive a volume density for the progenitors to be approximately $10^{-4} \mathrm{Mpc}^{-3}$. This is consistent to the volume density for SMGs at $z=1.5-3$ with $S_{870}>4 \mathrm{mJy}$ derived from Wardlow et al. (2011), which assumes the lifetime of the SMG phase to be $100 \mathrm{Myr}, 10$ times shorter than for DOGs. In this scenario, DOGs would have the same descendants as $z \sim 2$ SMGs, which are likely to be $2-3 L^{*}$ early-type galaxies (Wardlow et al. 2011; Hickox et al. 2012).

\section{CONCLUSIONS}

We use Herschel HerMES data in COSMOS to study the far-IR emission from DOGs. The main findings are as follows.
1. Out of 3077 DOGs, $51 \%$ are detected in Herschel $\left(S_{250} \geqslant\right.$ $3 \sigma=8 \mathrm{mJy})$. We use stacking to probe the remaining Herschel-undetected population and the stacked $S_{250}$ is on average a factor of $\sim 2$ fainter than the $250 \mu \mathrm{m}$ detection limit of $3 \sigma$.

2. The IR luminosity functions of DOGs and all $24 \mu \mathrm{m}$ sources with $S_{24} \geqslant 100 \mu \mathrm{Jy}$ at $z=1.5-2.5$ are calculated. The stacked IR luminosities provide significant contribution in the lowest Herschel-detected IR luminosity bin, causing an increase of $\sim 0.2$ dex. IR luminosities derived from extrapolating $24 \mu \mathrm{m}$ flux densities of CE01 templates are overestimated by a factor of two and in agreement with previous observations.

3. DOGs contribute $10 \%-30 \%$ to the overall SFR density of the universe and $30 \%$ to all $24 \mu \mathrm{m}$ galaxies with $S_{24} \geqslant$ $100 \mu \mathrm{Jy}$. We also note that when compared to the total DOG $\rho_{\text {SFR }}$, power-law (AGN-dominated) DOGs provide minor contributions. The $\rho_{\mathrm{SFR}}$ for DOGs and SMGs are comparable at $z \sim 2$; however, we note that DOGs are more numerous, with individually lower SFRs for DOGs than SMGs.

4. DOGs have a large scatter in the $\mathrm{SFR}-M_{*}$ plane, having sources in the starburst, main sequence, and more quiescent galaxy regimes. The observed phase of star formation for most DOGs is likely responsible for their observed stellar mass.

We thank the anonymous referee for useful comments that helped improve this paper. We thank Jason Melbourne for insightful discussions.

SPIRE has been developed by a consortium of institutes led by Cardiff University (UK) and including: University of Lethbridge (Canada); NAOC (China); CEA, LAM (France); IFSI, University of Padua (Italy); IAC (Spain); Stockholm Observatory (Sweden); Imperial College London, RAL, UCLMSSL, UKATC, Univ. Sussex (UK); and Caltech, JPL, NHSC, University of Colorado (USA). This development has been supported by national funding agencies: CSA (Canada); NAOC (China); CEA, CNES, CNRS (France); ASI (Italy); MCINN (Spain); SNSB (Sweden); STFC, UKSA (UK); and NASA (USA).

This research has made use of data from the HerMES project (http://hermes.sussex.ac.uk/). HerMES is a Herschel Key Programme utilizing Guaranteed Time from the SPIRE instrument team, ESAC scientists, and a mission scientist. The data presented in this paper will be released through the HerMES Database in Marseille, HeDaM (http://hedam.oamp.fr/HerMES/).

We our thankful to the COSMOS collaboration for granting us access to their catalogs and we gratefully acknowledge the contributions of the entire COSMOS team that have made this work possible. More information on the COSMOS survey is available at http://hermes.sussex.ac.uk/. This work is based (in part) on observations made with the Spitzer Space Telescope, which is operated by the Jet Propulsion Laboratory, California Institute of Technology under a contract with NASA. We acknowledge support from a GAANN fellowship (to J.A.C.), NSF CAREER AST-0645427 (A.C. and H.F.), and NASA funds to the US HerMES team through JPL. S.O. acknowledges support from the Science and Technology Facilities Council [grant number ST/I000976/1].

Some of the data (spectroscopic redshifts) presented here were obtained at the W. M. Keck Observatory, which is operated 
as a scientific partnership among the California Institute of Technology, the University of California, and the National Aeronautics and Space Administration. The Observatory was made possible by the generous financial support of the W. M. Keck Foundation. The authors wish to recognize and acknowledge the very significant cultural role and reverence that the summit of Mauna Kea has always had within the indigenous Hawaiian community. We are most fortunate to have the opportunity to conduct observations from this mountain.

Facilities: Herschel (SPIRE), Spitzer (IRAC, MIPS), Subaru (Suprime Cam), Keck:II (DEIMOS)

\section{REFERENCES}

Barger, A. J., Cowie, L. L., Sanders, D. B., et al. 1998, Natur, 394, 248 Béthermin, M., Dole, H., Beelen, A., \& Aussel, H. 2010, A\&A, 512, A78 Béthermin, M., Le Floc'h, E., Ilbert, O., et al. 2012, A\&A, 542, A58 Blain, A. W., Kneib, J.-P., Ivison, R. J., \& Smail, I. 1999, ApJL, 512, L87 Brodwin, M., Dey, A., Brown, M. J. I., et al. 2008, ApJL, 687, L65 Burgarella, D., Buat, V., Gruppioni, C., et al. 2013, A\&A, 554, A70 Bussmann, R. S., Dey, A., Armus, L., et al. 2012, ApJ, 744, 150 Bussmann, R. S., Dey, A., Lotz, J., et al. 2009, ApJ, 693, 750 Bussmann, R. S., Dey, A., Lotz, J., et al. 2011, ApJ, 733, 21 Capak, P., Aussel, H., Ajiki, M., et al. 2007, ApJS, 172, 99 Caputi, K. I., Lagache, G., Yan, L., et al. 2007, ApJ, 660, 97 Casey, C. M., Chapman, S. C., Beswick, R. J., et al. 2009, MNRAS, 399, 121 Casey, C. M., Chapman, S. C., Smail, I., et al. 2011, MNRAS, 411, 2739 Chapin, E. L., Pope, A., Scott, D., et al. 2009, MNRAS, 398, 1793 Chapman, S. C., Smail, I., Blain, A. W., \& Ivison, R. J. 2004, ApJ, 614, 671 Chary, R., \& Elbaz, D. 2001, ApJ, 556, 562

Daddi, E., Dickinson, M., Morrison, G., et al. 2007, ApJ, 670, 156

Dai, Y. S., Bergeron, J., Elvis, M., et al. 2012, ApJ, 753, 33

Dey, A., Soifer, B. T., Desai, V., et al. 2008, ApJ, 677, 943

Donley, J. L., Rieke, G. H., Pérez-González, P. G., Rigby, J. R., \& AlonsoHerrero, A. 2007, ApJ, 660, 167

Draine, B. T. 2003, ARA\&A, 41, 241

Eales, S. A., Raymond, G., Roseboom, I. G., et al. 2010, A\&A, 518, L23

Elbaz, D., Dickinson, M., Hwang, H. S., et al. 2011, A\&A, 533, A119

Elbaz, D., Hwang, H. S., Magnelli, B., et al. 2010, A\&A, 518, L29

Elvis, M., Wilkes, B. J., McDowell, J. C., et al. 1994, ApJS, 95, 1

Farrah, D., Afonso, J., Efstathiou, A., et al. 2003, MNRAS, 343, 585

Farrah, D., Lonsdale, C. J., Weedman, D. W., et al. 2008, ApJ, 677, 957

Fazio, G. G., Hora, J. L., Allen, L. E., et al. 2004, ApJS, 154, 10

Fiore, F., Grazian, A., Santini, P., et al. 2008, ApJ, 672, 94

Fiore, F., Puccetti, S., Brusa, M., et al. 2009, ApJ, 693, 447

Fu, H., Yan, L., Scoville, N. Z., et al. 2010, ApJ, 722, 653

Genzel, R., Tacconi, L. J., Rigopoulou, D., Lutz, D., \& Tecza, M. 2001, ApJ, 563,527

Griffin, M. J., Abergel, A., Abreu, A., et al. 2010, A\&A, 518, L3

Hatziminaoglou, E., Omont, A., Stevens, J. A., et al. 2010, A\&A, 518, L33

Hickox, R. C., Wardlow, J. L., Smail, I., et al. 2012, MNRAS, 421, 284

Hopkins, A. M., \& Beacom, J. F. 2006, ApJ, 651, 142

Houck, J. R., Soifer, B. T., Weedman, D., et al. 2005, ApJL, 622, L105

Hughes, D. H., Serjeant, S., Dunlop, J., et al. 1998, Natur, 394, 241

Hwang, H. S., Elbaz, D., Magdis, G., et al. 2010, MNRAS, 409, 75
Ilbert, O., Capak, P., Salvato, M., et al. 2009, ApJ, 690, 1236

Ilbert, O., Salvato, M., Le Floc'h, E., et al. 2010, ApJ, 709, 644 Kennicutt, R. C., Jr. 1998, ApJ, 498, 541

Kirkpatrick, A., Pope, A., Alexander, D. M., et al. 2012, ApJ, 759, 139

Komiyama, Y., Miyazaki, S., Yagi, M., et al. 2003, Proc. SPIE, 4841, 152

Le Floc'h, E., Aussel, H., Ilbert, O., et al. 2009, ApJ, 703, 222

Le Floc'h, E., Papovich, C., Dole, H., et al. 2005, ApJ, 632, 169

Levenson, L., Marsden, G., Zemcov, M., et al. 2010, MNRAS, 409, 83

Lilly, S. J., Le Fèvre, O., Renzini, A., et al. 2007, ApJS, 172, 70

Lonsdale, C. J., Farrah, D., \& Smith, H. E. 2006, in Ultraluminous Infrared Galaxies, ed. J. W. Mason (Heidelberg: Springer), 285

Lutz, D., Mainieri, V., Rafferty, D., et al. 2010, ApJ, 712, 1287

Lutz, D., Sturm, E., Tacconi, L. J., et al. 2008, ApJ, 684, 853

Magdis, G. E., Elbaz, D., Hwang, H. S., et al. 2010, MNRAS, 409, 22

Magliocchetti, M., Cirasuolo, M., McLure, R. J., et al. 2008, MNRAS, 383, 1131

Magnelli, B., Elbaz, D., Chary, R. R., et al. 2009, A\&A, 496, 57

Magnelli, B., Elbaz, D., Chary, R. R., et al. 2011, A\&A, 528, A35

Magnelli, B., Lutz, D., Berta, S., et al. 2010, A\&A, 518, L28

Magnelli, B., Lutz, D., Santini, P., et al. 2012, A\&A, 539, A155

Melbourne, J., Soifer, B. T., Desai, V., et al. 2012, AJ, 143, 125

Mullaney, J. R., Pannella, M., Daddi, E., et al. 2012, MNRAS, 419, 95

Narayanan, D., Dey, A., Hayward, C. C., et al. 2010, MNRAS, 407, 1701

Nordon, R., Lutz, D., Genzel, R., et al. 2012, ApJ, 745, 182

Nordon, R., Lutz, D., Shao, L., et al. 2010, A\&A, 518, L24

Oliver, S. J., Bock, J., Altieri, B., et al. 2012, MNRAS, 424, 1614

Papovich, C., Rudnick, G., Le Floc'h, E., et al. 2007, ApJ, 668, 45

Penner, K., Dickinson, M., Pope, A., et al. 2012, ApJ, 759, 28

Pérez-González, P. G., Rieke, G. H., Egami, E., et al. 2005, ApJ, 630, 82

Pilbratt, G. L., Riedinger, J. R., Passvogel, T., et al. 2010, A\&A, 518, L1

Polletta, M., Tajer, M., Maraschi, L., et al. 2007, ApJ, 663, 81

Pope, A., Bussmann, R. S., Dey, A., et al. 2008, ApJ, 689, 127

Richards, G. T., Lacy, M., Storrie-Lombardi, L. J., et al. 2006, ApJS, 166, 470

Rieke, G. H., Young, E. T., Engelbracht, C. W., et al. 2004, ApJS, 154, 25

Rodighiero, G., Cimatti, A., Gruppioni, C., et al. 2010, A\&A, 518, L25

Rodighiero, G., Daddi, E., Baronchelli, I., et al. 2011, ApJL, 739, L40

Roseboom, I. G., Oliver, S. J., Kunz, M., et al. 2010, MNRAS, 409, 48

Rowan-Robinson, M., Roseboom, I. G., Vaccari, M., et al. 2010, MNRAS, 409, 2

Sajina, A., Yan, L., Armus, L., et al. 2007, ApJ, 664, 713

Salvato, M., Hasinger, G., Ilbert, O., et al. 2009, ApJ, 690, 1250

Sanders, D. B., Salvato, M., Aussel, H., et al. 2007, ApJS, 172, 86

Schmidt, K.-H. 1978, AN, 299, 193

Scoville, N., Aussel, H., Brusa, M., et al. 2007, ApJS, 172, 1

Smail, I., Ivison, R. J., \& Blain, A. W. 1997, ApJL, 490, L5

Springel, V., Di Matteo, T., \& Hernquist, L. 2005, ApJL, 620, L79

Starikova, S., Berta, S., Franceschini, A., et al. 2012, ApJ, 751, 126

Swinyard, B. M., Ade, P., Baluteau, J.-P., et al. 2010, A\&A, 518, L4

Symeonidis, M., Vaccari, M., Berta, S., et al. 2013, MNRAS, 431, 2317

Veilleux, S., Rupke, D. S. N., Kim, D.-C., et al. 2009, ApJS, 182, 628

Wardlow, J. L., Smail, I., Coppin, K. E. K., et al. 2011, MNRAS, 415, 1479

Watabe, Y., Risaliti, G., Salvati, M., et al. 2009, MNRAS, 396, L1

Weedman, D. W., Le Floc'h, E., Higdon, S. J. U., Higdon, J. L., \& Houck, J. R. 2006, ApJ, 638, 613

Werner, M. W., Roellig, T. L., Low, F. J., et al. 2004, ApJS, 154, 1

Yan, L., Chary, R., Armus, L., et al. 2005, ApJ, 628, 604

Yan, L., Choi, P. I., Fadda, D., et al. 2004, ApJS, 154, 75

Yan, L., Sajina, A., Fadda, D., et al. 2007, ApJ, 658, 778 